\title{
Vortices in a Generalized Maxwell-Higgs Model with Visible and Hidden Sectors
}

\author{
D. Bazeia $\mathbb{D}^{1},{ }^{1}$ L. Losano, ${ }^{1}$ M. A. Marques $\mathbb{D},{ }^{1}$ and R. Menezes ${ }^{1,2}$ \\ ${ }^{1}$ Departamento de Física, Universidade Federal da Paraíba, 58051-970 João Pessoa, PB, Brazil \\ ${ }^{2}$ Departamento de Ciências Exatas, Universidade Federal da Paraíba, 58297-000 Rio Tinto, PB, Brazil
}

Correspondence should be addressed to D. Bazeia; dbazeia@gmail.com

Received 19 February 2019; Accepted 14 April 2019; Published 5 May 2019

Academic Editor: Rong-Gen Cai

Copyright (C) 2019 D. Bazeia et al. This is an open access article distributed under the Creative Commons Attribution License, which permits unrestricted use, distribution, and reproduction in any medium, provided the original work is properly cited. The publication of this article was funded by SCOAP ${ }^{3}$.

\begin{abstract}
We investigate the presence of vortices in generalized Maxwell-Higgs models with a hidden sector. The model engenders $U(1) \times U(1)$ symmetry, in a manner that the sectors are coupled via the visible magnetic permeability depending only on the hidden scalar field. We develop a first-order framework in which the hidden sector decouples from the visible one. We illustrate the results with two specific examples that give rise to the presence of vortices with internal structure.
\end{abstract}

\section{Introduction}

Topological structures appear in high energy physics in a variety of dimensions [1-3]. Among the mostly known ones, there are kinks, vortices, and monopoles, which are static configurations that appear in $(1,1),(2,1)$, and $(3,1)$ spacetime dimensions, respectively. These structures have been investigated for more than 40 years, and they find a myriad of applications in high energy and condensed matter physics [1-4].

Vortices, in particular, were firstly investigated by Helmholtz in [5] and are commonly found in fluid mechanics [6]. They also appear in condenser matter, in the study of type II superconductors under specific conditions [7] when one deals with the Ginzburg-Landau theory of superconductivity [8]. As it is known, the Ginzburg-Landau theory is nonrelativistic and the first relativistic model that supports vortex configurations was introduced by Nielsen and Olesen in [9]; see also [10, 11]. It consists of a Higgs field coupled to a gauge field with the Maxwell dynamics under a local $U(1)$ symmetry. These vortices are electrically neutral but have quantized magnetic flux.

They were largely investigated in several different contexts, including the case of generalized models; see [12-30]. In $[12,13]$, for instance, the presence of a generalized magnetic permeability in a model of the Maxwell-Higgs type allowed simulating properties of Chern-Simons vortices [31]. By using a similar model in [28], we have found the presence of compact vortices, which seems to map the magnetic field of an infinitely long solenoid. Due to the aforementioned works with generalized kinematics, we have introduced a first-order formalism to describe vortices in generalized models in [29] and a procedure to find analytic solutions in [30].

More recently, in [32], we have enlarged the $U(1)$ symmetry to accommodate a $Z_{2}$ symmetry, with the addition of a single neutral scalar field that interacts with the gauge field via the magnetic permeability. In this case, it was shown that the neutral field acts as a source to generate the vortex. In the past, a similar idea was developed, but with an $U(1) \times U(1)$ symmetry engendered by two gauge fields and two complex scalar fields interacting through an extension of the standard Higgs-like potential [33]. This model is of interest in the study of superconducting strings and has been used in several contexts, in particular in [3436] and in references therein. For instance, in [35] the authors describe the presence of novel solutions for non-Abelian cosmic strings and in [36] the study focuses on the critical behavior of magnetic field in a superconductor coupled to a superfluid, intending to simulate the core of neutrons stars, where superconducting protons are supposed to couple with superfluid neutrons. Among other possibilities, the $U(1)$ symmetry may be enlarged to accommodate other fields. 
In particular, in [37-39] the $U(1)$ symmetry is enlarged to become $U(1) \times S O(3)$, in which the $S O(3)$ symmetry is governed by the addition of some neutral scalar fields. Furthermore, in the recent work [40] we considered the symmetry $S U(2) \times Z_{2}$, extending the study of monopoles in the non-Abelian model considered $[41,42]$ to include a new neutral scalar field to produce magnetic monopoles with internal structure in the three-dimensional space.

Models with the $U(1) \times U(1)$ symmetry are useful to include the so-called hidden sector, which seems to be of current interest $[43,44]$ and may play a role in the study of dark matter [45-48]. This hidden (or dark) sector may be coupled to the visible one via the Higgs fields, as it appears in the Higgs portal $[49,50]$, in a way similar to the coupling that appeared before in [33-35]. Another possibility is to add the interaction of the gauge field strengths as in [51-55], with the two strength tensors coupled to each other. We can also enlarge the symmetry and consider $S U(2) \times U(1)$, as in [35], for instance, and also $S U(3) \times U(1)$, as investigated very recently in [56], to search for color-magnetic structures in dense quark matter, compatible with the interior of compact stars and able to produce detectable gravitational waves. In this work, however, we follow a different direction and consider the $U(1) \times U(1)$ symmetry, choosing the magnetic permeability of the visible sector to be driven by the scalar field of the hidden sector, without the coupling between the electromagnetic strength tensors. This is a novel possibility, which leads to results of current interest.

There are other interesting issues that suggest the study of topological structures in models described by the $U(1) \times U(1)$ symmetry, as in $[54,55]$ and in references therein. In this work, however, we deal with a relativistic model in $(2,1)$ spacetime dimension and, inspired by [9-11], we consider a model in which the first $U(1)$ portion describes the visible sector and the second accounts for the hidden sector. The system engenders two complex scalars of the Higgs type, the visible $\varphi$, and the hidden $\chi$ fields, with the coupling between the two sectors coming from a nonnegative function $P(|\chi|)$ that is controlled by the hidden scalar field. As we show below, the function $P(|\chi|)$ describes the magnetic permeability of the visible sector, and the main motivation for this is the fact that the dark matter seems to be always permeating the medium where the visible or baryonic matter appears to form galaxies in the Universe. Although we are dealing with a three-dimensional toy model, we believe that it is worth exploring this kind of coupling, since we still have no important insight on how the visible matter interacts with the dark component. Also, we will be searching for static vortexlike configurations at the classical level, intending to see how the topological structure of the hidden sector may modify the profile of the corresponding visible counterpart.

Dark matter is one among several problems that cannot be solved inside the Standard Model of particle physics, so the enhancement of the $U(1)$ symmetry to the case $U(1) \times U(1)$ is welcome to the study involving the visible and hidden sectors; see, e.g., the recent works $[57,58]$ and references therein, on several open problems in physics, in particular on dark matter. Moreover, since we are working at the classical level, the current study makes no progress on possible quantum features of the model. However, it can also be of interest to study pipelike vortices in two-component condensates [59] and also the interaction between vortices that can find applications in magnetic materials, for instance, if one changes the formulation in terms of the visible and hidden sectors to the case of hard and soft arrangements that appear in bimagnetic materials; see, e.g., $[60,61]$ and references therein. In this sense, the doubling of the $U(1)$ symmetry to the $U(1 \times U(1))$ case is of good use to investigate systems composed of two components condensates, not only in the case of visible and hidden sectors [51-54], but also in other contexts, such as the normal and superconductor components firstly studied in [33], the superconductor and superfluid coupling that is supposed to be present in the core of neutron stars [36], the study of pipelike vortex in twocomponent condensates [59], and the possibility of building two-component magnetic materials, with a hard magnetic component interacting with a soft magnetic component of the bimagnetic material $[60,61]$.

In order to introduce and investigate the $U(1) \times U(1)$ model, we organize the paper as follows: in Section 2, we present the model and develop the Bogomol'nyi procedure [11], to find first-order differential equations which are very relevant to describe stable vortex configurations. For the proposed model, we also show that the first-order framework acts to decouple the hidden sector from the visible one. This is an interesting result, and we can then use the hidden sector as a source to describe the visible sector. In Section 3, we illustrate the results with two examples, which give rise to vortices with features of current interest. We end the work in Section 4, where we add conclusions and perspectives for future works.

\section{The General Model}

We work in $(2,1)$ flat spacetime dimensions with the action $S=\int d^{3} x \mathscr{L}$, where the Lagrangian density is

$$
\begin{aligned}
\mathscr{L}= & -\frac{1}{4} P(|\chi|) F_{\mu \nu} F^{\mu \nu}-\frac{1}{4} Q(|\chi|) \mathscr{F}_{\mu \nu} \mathscr{F}^{\mu \nu}+\left|D_{\mu} \varphi\right|^{2} \\
& +\left|\mathscr{D}_{\mu} \chi\right|^{2}-V(|\varphi|,|\chi|) .
\end{aligned}
$$

Here $\varphi$ and $A_{\mu}$ are the visible complex scalar and gauge fields, and the corresponding hidden fields are $\chi$ and $\mathscr{A}_{\mu}$. As usual, $F_{\mu \nu}=\partial_{\mu} A_{\nu}-\partial_{\nu} A_{\mu}$ is the visible electromagnetic strength tensor, and $D_{\mu}=\partial_{\mu}+i e A_{\mu}$ stands for the covariant derivative. Their equivalent hidden counterparts are $\mathscr{F}_{\mu \nu}=$ $\partial_{\mu} \mathscr{A}_{\nu}-\partial_{\nu} \mathscr{A}_{\mu}$ and $\mathscr{D}_{\mu}=\partial_{\mu}+i q \mathscr{A}_{\mu}$. The potential is denoted by $V(|\varphi|,|\chi|)$ and may present terms that couple both the visible and hidden scalar fields. In principle, the explicit form of the potential is unknown, and the generalized magnetic permeabilities are controlled by $P(|\chi|)$ and $Q(|\chi|)$, which are nonnegative functions that depend exclusively on the field $\chi$. The above gauge invariant Lagrangian density engenders the $U(1) \times U(1)$ symmetry. The inclusion of functions of the scalar field multiplying the Maxwell term is also present in models related to holography; see, e.g., [62, 63] for two distinct possibilities, used to describe an holographic insulator model 
with nonsingular zero temperature infrared geometry [62] and the electric charge transport in a strongly coupled quarkgluon plasma [63].

In our model, the visible and hidden sectors are coupled through $P(|\chi|)$, i.e., the hidden scalar field controls the magnetic permeability of the visible sector. This is a different approach from the one considered in [55], where the coupling was done with the electromagnetic strength tensors, through the term $F_{\mu \nu} \mathscr{F}^{\mu \nu}$, which we are not considering in the current work. Here, we use the metric tensor $\eta_{\mu \nu}=(1,-1,-1)$ and the natural units $\hbar=c=1$.

By varying the action with respect to the fields, we get that the equations of motion associated with the Lagrangian density (1) are

$$
\begin{aligned}
& D_{\mu} D^{\mu} \varphi=-\frac{\varphi}{2|\varphi|} V_{|\varphi|}, \\
& \mathscr{D}_{\mu} \mathscr{D}^{\mu} \chi \\
& \quad=-\frac{\chi}{2|\chi|}\left(\frac{P_{|\chi|}}{4} F_{\mu \nu} F^{\mu \nu}+\frac{Q_{|\chi|}}{4} \mathscr{F}_{\mu \nu} \mathscr{F}^{\mu \nu}+V_{|\chi|}\right), \\
& \partial_{\mu}\left(P F^{\mu \nu}\right)=J^{\nu}, \\
& \partial_{\mu}\left(Q \mathscr{F}^{\mu \nu}\right)=\mathscr{J}^{\nu},
\end{aligned}
$$

where the currents are $J_{\mu}=i e\left(\bar{\varphi} D_{\mu} \varphi-\varphi \overline{D_{\mu} \varphi}\right)$ and $\mathscr{J}_{\mu}=$ $i q\left(\bar{\chi} \mathscr{D}_{\mu} \chi-\chi \overline{D_{\mu}} \chi\right)$. Also, we have used the notation $V_{|\varphi|}=$ $\partial V / \partial|\varphi|, V_{|\chi|}=\partial V / \partial|\chi|$ and so on. By setting $\nu=0$ in (2c) and (2d) and considering static field configurations, one can show the Gauss' laws are identities for $A_{0}=0$ and for $\mathscr{A}_{0}=0$. In this case, the solutions are electrically neutral since the electric charges vanish.

Invariance under spacetime translations $x^{\mu} \longrightarrow x^{\mu}+b^{\mu}$, with $b^{\mu}$ constant, lead to the conserved energy-momentum tensor

$$
\begin{aligned}
T_{\mu \nu}= & P F_{\mu \lambda} F_{\nu}^{\lambda}+Q \mathscr{F}_{\mu \lambda} \mathscr{F}_{\nu}^{\lambda}+\overline{D_{\mu} \varphi} D_{\nu} \varphi+\overline{D_{\nu} \varphi} D_{\mu} \varphi \\
& +\overline{\mathscr{D}_{\mu} \chi} \mathscr{D}_{\nu} \chi+\overline{\mathscr{D}}_{\nu} \chi \mathscr{D}_{\mu} \chi-\eta_{\mu \nu} \mathscr{L} .
\end{aligned}
$$

To search for vortexlike solutions, we consider static configurations and the usual ansatz

$$
\begin{aligned}
\varphi & =g(r) e^{i n \theta}, \\
\chi & =h(r) e^{i k \theta}, \\
\vec{A} & =\frac{\widehat{\theta}}{e r}[n-a(r)], \\
\overrightarrow{\mathscr{A}} & =\frac{\widehat{\theta}}{q r}[k-c(r)],
\end{aligned}
$$

in which $n$ and $k$ are nonvanishing integer numbers that control the vorticity of the visible and hidden solutions, respectively. Although this is not the most general situation, the above cylindrically symmetric ansatz for the fields is largely used in the search for vortex solutions in planar systems; see, e.g., $[9-11,54,55]$ and references therein. Also, when $P(|\chi|)=1$ the visible and hidden sectors decouple, and the expressions in (4) lead us back to the standard situation. The functions $a(r), c(r), g(r)$, and $h(r)$ obey the boundary conditions

$$
\begin{gathered}
g(0)=0, \\
h(0)=0, \\
a(0)=n, \\
c(0)=k, \\
g(\infty)=v, \\
h(\infty)=w, \\
a(\infty)=0, \\
c(\infty)=0 .
\end{gathered}
$$

Here, $v$ and $w$ are parameters that control the asymptotic behavior of the functions $g(r)$ and $h(r)$. Considering (4), the visible and hidden magnetic fields are giving by, respectively,

$$
\begin{aligned}
B & =-F^{12}=-\frac{a^{\prime}}{e r} \\
\text { and } \mathscr{B} & =-\mathscr{F}^{12}=-\frac{c^{\prime}}{q r},
\end{aligned}
$$

where the prime stands for the derivative with respect to $r$. By using this, one can show the magnetic fluxes are quantized

$$
\begin{gathered}
\Phi_{(B)}=2 \pi \int r d r B=\frac{2 \pi}{e} n, \\
\Phi_{(\mathscr{B})}=2 \pi \int r d r \mathscr{B}=\frac{2 \pi}{q} k .
\end{gathered}
$$

These results allow that we introduce two topological currents, one in the visible sector, defined by $J_{T}^{\mu}=\varepsilon^{\mu \nu \lambda} \partial_{\lambda} A_{v}$, and the other in the hidden sector, defined by $\mathcal{F}_{T}^{\mu}=\varepsilon^{\mu \nu \lambda} \partial_{\lambda} \mathscr{A}_{\gamma}$; they are conserved and describe conserved charges that are directly identified with the corresponding magnetic fluxes

$$
\begin{aligned}
& Q_{T}=2 \pi \int r d r J_{T}^{0}=\Phi_{(B)}, \\
& Q_{T}=2 \pi \int r d r \mathscr{F}_{T}^{0}=\Phi_{(\mathscr{B})} .
\end{aligned}
$$

The above results inform us that the suggested solutions are topologically protected against decay into the elementary excitations of the system.

We use (4) to rewrite the equations of motion (2a), (2b), $(2 \mathrm{c})$, and $(2 \mathrm{~d})$ in the form 


$$
\begin{aligned}
& \frac{1}{r}\left(r g^{\prime}\right)^{\prime}-\frac{a^{2} g}{r^{2}}-\frac{1}{2} V_{|\varphi|}=0, \\
& \frac{1}{r}\left(r h^{\prime}\right)^{\prime}-\frac{c^{2} h}{r^{2}}-\frac{1}{2}\left(P_{|\chi|} \frac{a^{\prime 2}}{2 e^{2} r^{2}}+Q_{|\chi|} \frac{c^{\prime 2}}{2 q^{2} r^{2}}+V_{|\chi|}\right) \\
& \quad=0 \\
& r\left(P \frac{a^{\prime}}{e r}\right)^{\prime}-2 e a g^{2}=0, \\
& r\left(Q \frac{c^{\prime}}{q r}\right)^{\prime}-2 q c h^{2}=0 .
\end{aligned}
$$

Using (4), the nonvanishing components of energymomentum tensor (3) become

$$
\begin{aligned}
T_{00}= & P \frac{a^{\prime 2}}{2 e^{2} r^{2}}+Q \frac{c^{\prime 2}}{2 q^{2} r^{2}}+g^{\prime 2}+\frac{a^{2} g^{2}}{r^{2}}+h^{\prime 2}+\frac{c^{2} h^{2}}{r^{2}} \\
& +V, \\
T_{12}= & \left(g^{\prime 2}-\frac{a^{2} g^{2}}{r^{2}}+h^{\prime 2}-\frac{c^{2} h^{2}}{r^{2}}\right) \sin (2 \theta), \\
T_{11}= & P \frac{a^{\prime 2}}{2 e^{2} r^{2}}+Q \frac{c^{\prime 2}}{2 q^{2} r^{2}}+\left(g^{\prime 2}+h^{\prime 2}\right)\left(2 \cos ^{2} \theta-1\right) \\
& +\left(\frac{a^{2} g^{2}}{r^{2}}+\frac{c^{2} h^{2}}{r^{2}}\right)\left(2 \sin ^{2} \theta-1\right)-V, \\
T_{22}= & P \frac{a^{\prime 2}}{2 e^{2} r^{2}}+Q \frac{c^{\prime 2}}{2 q^{2} r^{2}}+\left(g^{\prime 2}+h^{\prime 2}\right)\left(2 \sin ^{2} \theta-1\right) \\
& +\left(\frac{a^{2} g^{2}}{r^{2}}+\frac{c^{2} h^{2}}{r^{2}}\right)\left(2 \cos ^{2} \theta-1\right)-V .
\end{aligned}
$$

In the above equations, we identify the energy density as $\rho=$ $T_{00}$ and the stress tensor as $T_{i j}$. The equations of motion (9a), (9b), (9c), and (9d) present coupling between the functions and are of second order. In order to simplify the problem, it is of interest to find a first-order formalism for the model. This can be done by using (10a) to develop the Bogomol'nyi procedure [11]. After some algebraic manipulations, we can write

$$
\begin{aligned}
\rho= & \frac{P}{2}\left(\frac{a^{\prime}}{e r}+\frac{e\left(v^{2}-g^{2}\right)}{P}\right)^{2} \\
& +\frac{Q}{2}\left(\frac{c^{\prime}}{q r}+\frac{q\left(w^{2}-h^{2}\right)}{Q}\right)^{2}+\left(g^{\prime}-\frac{a g}{r}\right)^{2} \\
& +\left(h^{\prime}-\frac{c h}{r}\right)^{2}+V-\frac{e^{2}}{2} \frac{\left(v^{2}-g^{2}\right)^{2}}{P}
\end{aligned}
$$

$$
\begin{aligned}
& +\frac{q^{2}}{2} \frac{\left(w^{2}-h^{2}\right)^{2}}{Q} \\
& -\frac{1}{r}\left(a\left(v^{2}-g^{2}\right)+c\left(w^{2}-h^{2}\right)\right)^{\prime} .
\end{aligned}
$$

As we stated before, the potential is in principle arbitrary. However, motivated by the Bogomol'nyi procedure [11], the choice

$$
V(|\varphi|,|\chi|)=\frac{e^{2}}{2} \frac{\left(v^{2}-|\varphi|^{2}\right)^{2}}{P(|\chi|)}+\frac{q^{2}}{2} \frac{\left(w^{2}-|\chi|^{2}\right)^{2}}{Q(|\chi|)}
$$

allows that one writes the energy in the form

$$
\begin{aligned}
E= & 2 \pi \int_{0}^{\infty} r d r \frac{P}{2}\left(\frac{a^{\prime}}{e r}+\frac{e\left(v^{2}-g^{2}\right)}{P}\right)^{2} \\
& +2 \pi \int_{0}^{\infty} r d r \frac{Q}{2}\left(\frac{c^{\prime}}{q r}+\frac{q\left(w^{2}-h^{2}\right)}{Q}\right)^{2} \\
& +2 \pi \int_{0}^{\infty} r d r\left(g^{\prime}-\frac{a g}{r}\right)^{2} \\
& +2 \pi \int_{0}^{\infty} r d r\left(h^{\prime}-\frac{c h}{r}\right)^{2}+E_{B},
\end{aligned}
$$

where

$$
\begin{aligned}
E_{B} & =2 \pi \int_{0}^{\infty} d r\left(a\left(v^{2}-g^{2}\right)+c\left(w^{2}-h^{2}\right)\right)^{\prime} \\
& =2 \pi\left(|n| v^{2}+|k| w^{2}\right) .
\end{aligned}
$$

Although the choice (12) is not necessary, it leads us to (13), which unveils the interesting possibility: the first four terms are all nonnegative, so we see that the energy is bounded to the value $E=E_{B}$, if the solutions obey the first-order equations

$$
\begin{aligned}
g^{\prime} & =\frac{a g}{r}, \\
-\frac{a^{\prime}}{e r} & =\frac{e\left(v^{2}-g^{2}\right)}{P(h)},
\end{aligned}
$$

for $a(r)$ and $g(r)$ controlling the gauge and scalar fields in the visible sector, and

$$
\begin{aligned}
h^{\prime} & =\frac{c h}{r}, \\
-\frac{c^{\prime}}{q r} & =\frac{q\left(w^{2}-h^{2}\right)}{Q(h)},
\end{aligned}
$$

for $c(r)$ and $h(r)$ controlling the gauge and scalar in the hidden sector. Therefore, we have obtained four first-order equations to study the problem. One can show that they 
satisfy the equations of motion (9a), (9b), (9c), and (9d) with the potential (12). An interesting fact is that solutions of the first-order equations are endowed with minimum energy, so they are stable according to the classical result of Bogomol'nyi [11] and also, they are compatible with the stressless condition, $T_{i j}=0$, which assures stability of the solutions under rescaling; see [29, 64, 65]. Notice that the first-order equations (16a) and (16b) are decoupled from the ones for the visible sector in (15a) and (15b). Therefore, we firstly solve the hidden sector to see how it modifies the visible one by choosing $P(|\chi|)$.

Before doing that, however, we notice that the presence of the first-order equations (16a) and (16b) and (15a) and (15b) allows us to write the energy density (10a) in the form $\rho=$ $\rho_{\text {hidden }}+\rho_{\text {visible }}$, where

$$
\begin{aligned}
& \rho_{\text {hidden }}=Q(h) \frac{c^{\prime 2}}{q^{2} r^{2}}+2 h^{\prime 2}, \\
& \rho_{\text {visible }}=P(h) \frac{a^{\prime 2}}{e^{2} r^{2}}+2 g^{\prime 2}
\end{aligned}
$$

indicate the contribution of the visible and hidden sectors, respectively. We also highlight the fact that, from (14), the energy of the visible and hidden sectors are independent and fixed, despite the presence of the functions $P(|\chi|)$ and $Q(|\chi|)$. For the visible sector, we have $E_{\text {visible }}=2 \pi v^{2}|n|$ and for the hidden one, $E_{\text {hidden }}=2 \pi w^{2}|k|$.

Another feature of the above procedure, which can be seen from the potential in (12) and the first-order equations (15a) and (15b) and (16a) and (16b), is the presence of spontaneous symmetry breaking (SSB) in both the visible and hidden sectors. The presence of SSB in the hidden sector is necessary to give rise to vortex in the sector, and the first term in (12) induces SSB in the visible sector and is mandatory to generate the first-order equations that attain stable minima energy configurations. In particular, for $P(|\chi|)=1$ and $Q(|\chi|)=1$, one gets to the case of two uncoupled and similar $(e \longleftrightarrow q$ and $v \longleftrightarrow w)$ Maxwell-Higgs models, each one with its own Nielsen-Olesen vortex configuration [8]. To keep the two sectors coupled one has to make $P(|\chi|)$ nontrivial, and this we discuss in the next section. Moreover, one has to choose $P(|\chi|)$ and $Q(|\chi|)$ carefully, because they have to lead to first-order equations (15a) and (15b) and (16a) and (16b) that support solutions compatible with the boundary conditions (5a) and (5b).

\section{Some Specific Models}

Let us now illustrate our findings with two distinct examples. Before doing so, we can simplify the problem by rescaling the fields as

$$
\begin{aligned}
\varphi & \longrightarrow v \varphi, \\
\chi & \longrightarrow v \chi, \\
A_{\mu} & \longrightarrow v A_{\mu} \\
\text { and } \mathscr{A}_{\mu} & \longrightarrow v \mathscr{A}_{\mu},
\end{aligned}
$$

and taking $r \longrightarrow r / e v$ and $\mathscr{L} \longrightarrow e^{2} v^{4} \mathscr{L}$. This leads us to work with dimensionless quantities. We also consider $e=1$ and $v=1$ and understand the parameter $q$ and $w$ as the ratio of the respective constants $(q / e)$ and $(w / v)$ of the hidden and visible sectors.

3.1. First Example. Considering the hidden sector to be controlled by $Q(|\chi|)=1$, its first-order equations (16a) and (16b) take the form

$$
\begin{aligned}
h^{\prime} & =\frac{c h}{r}, \\
-\frac{c^{\prime}}{q r} & =q\left(w^{2}-h^{2}\right),
\end{aligned}
$$

which admits vortexlike solutions of the Nielsen-Olesen type. Unfortunately, the analytical solutions $a(r)$ and $h(r)$ are currently unknown. Therefore, one has to use numerical methods to find them. Since their profiles are well known (see $[9,10])$, we will not display them here.

We then go on and investigate how the hidden fields modify the visible sector. We take the choice

$$
P(|\chi|)=\frac{1}{\left(1-\beta|\chi|^{2}\right)^{2}},
$$

where $\beta$ is a nonnegative, dimensionless parameter. Notice that $\beta$ controls how strongly the hidden scalar field modifies the magnetic permeability of the visible sector. The case $\beta=0$ leads to $P(|\chi|)=1$, which decouples the sectors. The above function makes the potential in (12) to be written as

$$
\begin{aligned}
V(|\varphi|,|\chi|)= & \frac{1}{2}\left(1-|\varphi|^{2}\right)^{2}\left(1-\beta|\chi|^{2}\right)^{2} \\
& +\frac{q^{2}}{2}\left(w^{2}-|\chi|^{2}\right)^{2} .
\end{aligned}
$$

From (16a) and (16b), we get that the first-order equations for the visible sector are

$$
\begin{aligned}
g^{\prime} & =\frac{a g}{r}, \\
-\frac{a^{\prime}}{r} & =\left(1-g^{2}\right)\left(1-\beta h^{2}\right)^{2} .
\end{aligned}
$$

It is straightforward to see that the case $\beta=0$ leads to Nielsen-Olesen solutions, which are very similar to the ones of the hidden sector, except for the constants $q$ and $w$. The parameter $\beta$ plays an important role in the model. One can show that $a^{\prime}(r)$ vanishes for $h^{2}=1 / \beta$. We then expect to see a region in which $a(r)$ is approximately uniform. Since $0 \leq h(r)<1$, this feature appears only for $\beta>1$, which is the range that we will consider here.

In Figure 1, we plot the solutions of the above equations, the visible magnetic field (6) and the energy density (10a) for some values of $\beta$ and $q, w=1$. As $\beta$ increases, the solutions, the magnetic field, and the energy density shrink. The solution $a(r)$, in particular, presents a region in which it is 

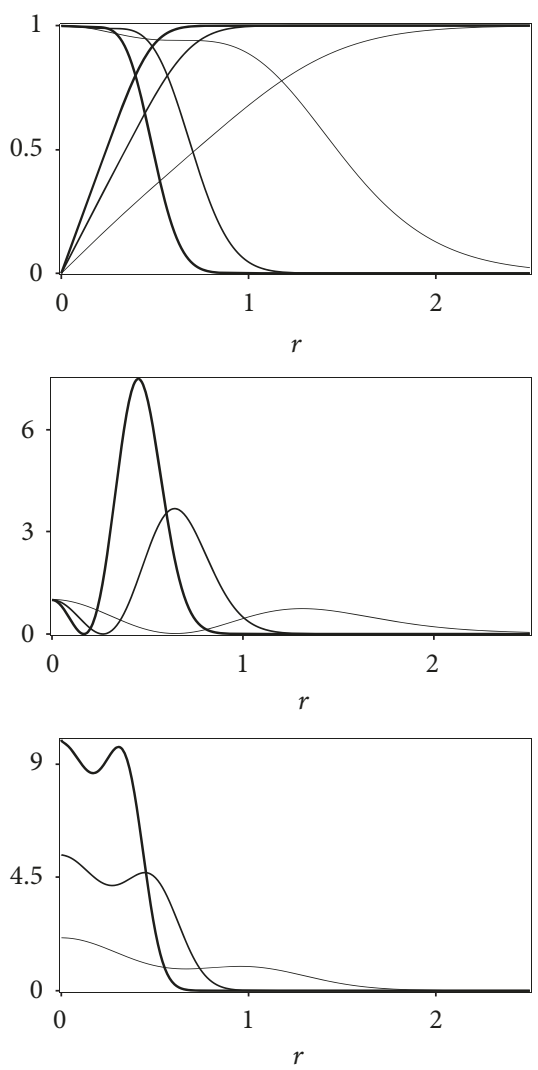

Figure 1: The solutions $a(r)$ (descending lines) and $g(r)$ (ascending lines) of (22a) and (22b) (top), the magnetic field (middle), and the energy density (bottom) of the visible sector for $q, w=1$ and $\beta=4,20$ and 50. The thickness of the lines increases with $\beta$.

approximately uniform, as expected. This region is very wide for $\beta \approx 1$ and tends to shrink and to become closer to the origin as we increase $\beta$. Due to this behavior in the solution, the magnetic field presents a splitting. This also happens, in a more subtle manner, with the energy density. Therefore, the presence of the hidden sector generates an internal structure to the vortex engendered by the visible sector of the model. It is worth commenting that these features do not affect the magnetic flux and the energy of the vortex, which remain unchanged, given by (7) and (14).

The presence of an internal structure in the quantities related to the visible sector motivated us to plot the magnetic field and the energy density in the plane. It can be seen in Figure 2.

3.2. Second Example. We now make a modification in the magnetic permeability of the hidden sector and suggest that it is driven by the function

$$
Q(|\chi|)=\frac{w^{2}}{2\left(w^{2}-|\chi|^{2}\right)}
$$

According to the boundary conditions in (5a) and (5b), $|\chi| \in[0, w]$; thus, $Q(|\chi|)$ is nonnegative where the solution exists. From (16a) and (16b), we get the following first-order equations for the hidden sector:

$$
\begin{aligned}
h^{\prime} & =\frac{c h}{r}, \\
-\frac{c^{\prime}}{q r} & =\frac{2 q}{w^{2}}\left(w^{2}-h^{2}\right)^{2} .
\end{aligned}
$$

In this case, we have been able to find their analytical solutions. They are given by

$$
\begin{gathered}
c(r)=\frac{1}{1+(q w r)^{2}} \\
\text { and } h(r)=\frac{q w^{2} r}{\sqrt{1+(q w r)^{2}}} .
\end{gathered}
$$

The above expression for $c(r)$ allows us to calculate the magnetic field associated with the hidden gauge field from (6), which is

$$
\mathscr{B}=\frac{2 q w^{2}}{\left(1+(q w r)^{2}\right)^{2}} .
$$

The energy density of the hidden sector in (17a) may be also found explicitly

$$
\rho_{\text {hidden }}=\frac{2 q^{2} w^{4}\left(3+(q w r)^{2}\right)}{\left(1+(q w r)^{2}\right)^{4}} .
$$




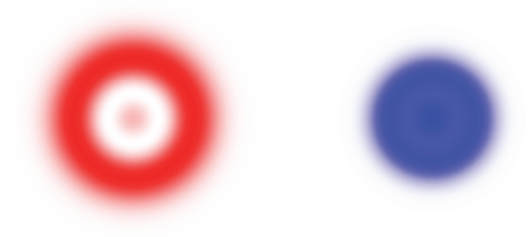

Figure 2: The magnetic field (left) and the energy density (right) of the solutions of (22a) and (22b) for the visible sector, with $q, w=1$ and $\beta=20$. The darkness of the colors is directly related to the intensity of the quantities.

Since the profiles of the functions in (25)-(27) are very similar to the ones that appear in the well-known Nielsen-Olesen case, they are not depicted here, although they are found analytically and easy to display.

We then investigate how the solutions (25) modify the visible sector by considering its magnetic permeability to be governed by the function

$$
P(|\chi|)=\frac{w^{2}}{\beta|\chi|^{2}}
$$

with $\beta$ real and positive. By doing so, the potential in (12) can be written as

$$
V(|\varphi|,|\chi|)=\frac{\beta}{2}\left(1-|\varphi|^{2}\right)^{2}|\chi|^{2}+\frac{q^{2}}{w^{2}}\left(w^{2}-|\chi|^{2}\right)^{3} .
$$

In this case, the first-order equations for the visible sector are

$$
\begin{aligned}
g^{\prime} & =\frac{a g}{r}, \\
-\frac{a^{\prime}}{r} & =\beta\left(1-g^{2}\right) \frac{q^{2} w^{2} r^{2}}{1+q^{2} w^{2} r^{2}} .
\end{aligned}
$$

The solutions of the above equations, the visible magnetic field (6), and energy density (10a) are depicted in Figure 3. Here, we highlight that the presence of the analytical solutions in (25) allows us to show that the derivative of the solution $a(r)$ vanishes at the origin. This makes the solution $a(r)$ approximately uniform in the neighborhood of this point. So, the solutions $a(r)$ present a plateau around the origin that leads to a hole in the magnetic field around the center of the vortex. This behavior also appears in the energy density, although in a subtle manner. As one can see here, the parameter $\beta$ is associated with the width of the solution and to the deepness of the valley around the origin in the magnetic field.

This model engenders a distinct behavior from the previous one in a qualitative manner, presenting a hole in magnetic field near the origin. The new features supported by our models motivated us to depict the magnetic field and the energy density for the solutions of (30a) and (30b) of the visible sector in the plane. It can be seen in Figure 4. As it was mentioned before, the presence of a hole is stronger in the magnetic field than in the energy density.

\section{Conclusions}

In this work, we studied vortex structures in generalized Maxwell-Higgs models with visible and hidden sectors. The models considered in this work contain two additional functions, $P(|\chi|)$ and $Q(|\chi|)$, that control the magnetic permeability of the visible and hidden sectors, respectively. The interaction between the two sectors is controlled by the hidden scalar field which appears in the function $P(|\chi|)$.

We have chosen a specific form of the potential, which allowed for the Bogomol'nyi procedure to work out and for the presence of first-order differential equations that solve the equations of motion. The procedure has shown that the first-order equations of the hidden sector decouple from the visible one and could be solved independently. In this sense, the hidden sector acts as a source for the solutions in the visible sector. By taking specific forms for the magnetic permeabilities, we have found that the hidden charged scalar field affects the visible vortex configuration, generating an internal structure to it. In the first example, the magnetic field presents an apparent valley outside the origin that seems to connect two separated structures. The effect is less evident in the energy density, although it is also there. In the second example, the vortex presents a magnetic field with a hole around the origin. Surprisingly, the energies and fluxes of the hidden and visible vortices are fixed by the boundary conditions, and they are independent from each other. Moreover, they do not depend on the specific form we choose for the magnetic permeabilities to construct the system. As a particularly interesting result, we could find a specific function $Q(|\chi|)$ which allowed us to describe the vortex solution of the hidden sector, and the corresponding magnetic field and energy density, analytically.

Here we have discarded the coupling between the two electromagnetic strength tensors, so an issue to be further examined would be to add this kind of coupling. Another possibility is to generalize the covariant derivative terms, as suggested before in [30]. Since the model examined above attains the Bogomol'nyi bound, it appears to be the bosonic portion of a lager, supersymmetric theory, and this is presently under consideration, to see how supersymmetry is working to lead us to the first-order equations.

We are also thinking of enlarging the model to accommodate other symmetries, such as the $S U(2) \times U(1)$ symmetry, to explore the presence of solutions within the non-Abelian context examined before in [35], and also the $S U(3) \times U(1)$ 

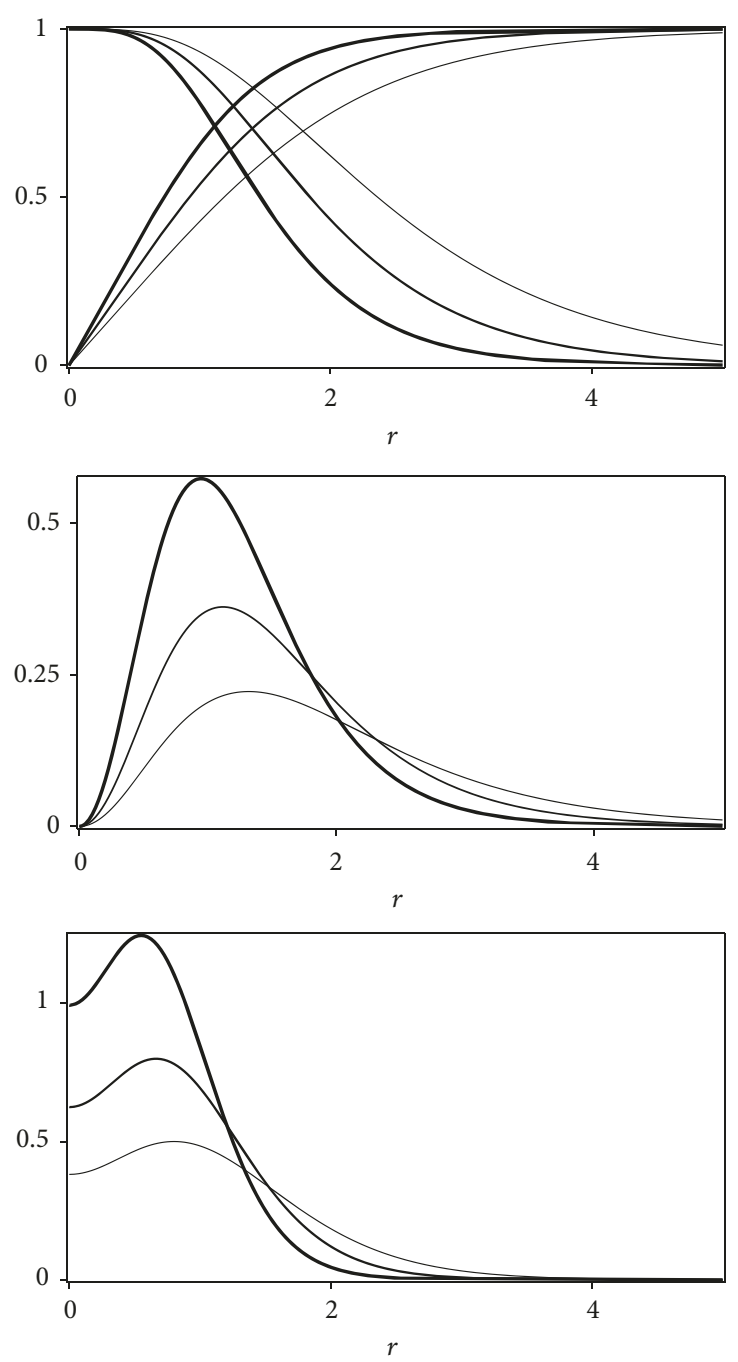

Figure 3: The solutions $a(r)$ (descending lines) and $g(r)$ (ascending lines) of (30a) and (30b) (top), the magnetic field (middle), and the energy density (bottom) of the visible sector for $q, w=1$ and $\beta=0.5,1$ and 2 . The thickness of the lines increases with $\beta$.

FIGURE 4: The magnetic field (left) and the energy density (right) of the solutions of (30a) and (30b) for the visible sector, with $q$, $w=1$ and $\beta=2$. The darkness of the colors is as in Figure 2 .

symmetry, to investigate color-magnetic structures in the dense quark matter scenario explored recently in [56]. The presence of non-Abelian symmetries makes the problem much harder, so the search for first-order differential equations that solve the equations of motion is of current interest and would be welcome.
Another possibility is to use the same $U(1) \times U(1)$ symmetry, but now changing the Maxwell dynamics of the gauge field in the hidden sector by the Chern-Simons one, to see how the Chern-Simons vortex may modify the MaxwellHiggs vortex in the visible sector. We hope to report on some of these issues in the near future. 


\section{Data Availability}

We inform that the calculations in the work are analytical and numerical. The analytical calculations are fully explained in the text of the manuscript. The numerical calculations are also standard and consist in solving differential equations numerically. The numerical and analytical calculations that we did in the manuscript are all well established and can be checked by others with no further difficulties.

\section{Conflicts of Interest}

The authors declare that they have no conflicts of interest.

\section{Acknowledgments}

We would like to acknowledge the Brazilian agency $\mathrm{CNPq}$ for partial financial support. D. Bazeia acknowledges support from Grant 306614/2014-6, L. Losano acknowledges support from Grant 303824/2017-4, M. A. Marques acknowledges support from Grant 155551/2018-3, and R. Menezes acknowledges support from Grant 306504/2018-9.

\section{References}

[1] A. Vilenkin and E. P. Shellard, Cosmic Strings and Other Topological Defects, Cambridge University Press, Cambridge, UK, 1994

[2] N. Manton and P. Sutcliffe, Topological Solitons, Cambridge University Press, Cambridge, UK, 2004.

[3] E. J. Weinberg, Classical Solutions in Quantum Field Theory: Solitons and Instantons in High Energy Physics, Cambridge University Press, 2012.

[4] E. Fradkin, Field Theories of Condensed Matter Physics, Cambridge University Press, Cambridge, UK, 2013.

[5] H. Helmholtz, "Über integrale der hydrodynamischen Gleichungen, welche den wirbelbewegungen entsprechen," Journal für die Reine und Angewandte Mathematik. [Crelle's Journal], vol. 55, pp. 25-55, 1858.

[6] P. G. Saffman, Vortex Dynamics, Cambridge University Press, Cambridge, UK, 1992.

[7] A. A. Abrikosov, "On the magnetic properties of superconductors of the second group," Zhurnal Eksperimentalnoi $i$ Teoreticheskoi Fiziki, vol. 32, p. 1442, 1957, Soviet Physics-JETP, vol. 5, p. 1174, 1957.

[8] V. L. Ginzburg and L. D. Landau, "On the theory of superconductivity," Zhurnal Eksperimentalnoi i Teoreticheskoi Fiziki, vol. 20, p. 1064, 1950.

[9] H. B. Nielsen and P. Olesen, "Vortex-line models for dual strings," Nuclear Physics B, vol. 61, pp. 45-61, 1973.

[10] H. J. de Vega and F. A. Schaposnik, "Classical vortex solution of the Abelian Higgs model," Physical Review D: Particles, Fields, Gravitation and Cosmology, vol. 14, no. 4, pp. 1100-1106, 1976.

[11] E. Bogomol'nyi, “The stability of classical solutions," Soviet Journal of Nuclear Physics, vol. 24, pp. 449-454, 1976.

[12] J. Lee and S. Nam, "Bogomol'nyi equations of Chern-Simons Higgs theory from a generalized abelian Higgs model," Physics Letters B, vol. 261, no. 4, pp. 437-442, 1991.
[13] D. Bazeia, "Vortices in a generalized Higgs model," Physical Review D: Particles, Fields, Gravitation and Cosmology, vol. 46, no. 4, pp. 1879-1881, 1992.

[14] E. Babichev, "Gauge k-vortices," Physical Review D: Particles, Fields, Gravitation and Cosmology, vol. 77, Article ID 065021, 2008.

[15] C. Adam, P. Klimas, J. Sánchez-Guillén, and A. Wereszczyński, "Compact gauge K vortices," Journal of Physics A: Mathematical and Theoretical, vol. 42, no. 13, Article ID 135401, 19 pages, 2009.

[16] D. Bazeia, E. da Hora, C. dos Santos, and R. Menezes, "BPS solutions to a generalized Maxwell-Higgs model," The European Physical Journal C, vol. 71, no. 12, pp. 1-9, 2011.

[17] A. N. Atmaja, H. S. Ramadhan, and E. da Hora, "More on Bogomol'nyi equations of three-dimensional generalized Maxwell-Higgs model using on-shell method," Journal of High Energy Physics, vol. 2016, no. 2, article no. 117, 2016.

[18] R. Casana, A. Cavalcante, and E. da Hora, "Self-dual configurations in Abelian Higgs models with k-generalized gauge field dynamics," Journal of High Energy Physics, vol. 2016, no. 12, article no. 51, 2016.

[19] A. N. Atmaja, "A method for BPS equations of vortices," Physics Letters B, vol. 768, pp. 351-358, 2017.

[20] E. Moreno, C. Núñez, and F. A. Schaposnik, "Electrically charged vortex solution in Born-Infeld theory," Physical Review D: Particles, Fields, Gravitation and Cosmology, vol. 58, no. 2, Article ID 025015, 1998.

[21] A. Alonso-Izquierdo, W. G. Fuertes, and J. M. Guilarte, "Two species of vortices in massive gauged non-linear sigma models," Journal of High Energy Physics, vol. 2015, no. 2, article no. 139, 2015.

[22] C. P. Burgess, R. Diener, and M. Williams, "The gravity of dark vortices: effective field theory for branes and strings carrying localized flux," Journal of High Energy Physics, vol. 49, no. 11, article no. 1511, 2015.

[23] X. Han and Y. Yang, "Magnetic impurity inspired Abelian Higgs vortices," Journal of High Energy Physics, vol. 2016, no. 2, article no. 46, 2016.

[24] P. Forgács and Á. Lukács, "Vortices and magnetic bags in Abelian models with extended scalar sectors and some of their applications," Physical Review D: Particles, Fields, Gravitation and Cosmology, vol. 94, no. 12, Article ID 125018, 2016.

[25] J. Chagoya and G. Tasinato, "Galileon Higgs vortices," Journal of High Energy Physics, vol. 1602, no. 2, article no. 63, 2016.

[26] C. Adam, J. M. Speight, and A. Wereszczynski, "Volume of a vortex and Bradlow bound," Physical Review D: Particles, Fields, Gravitation and Cosmology, vol. 95, no. 11, Article ID 116007, 19 pages, 2017.

[27] D. Bazeia, M. A. Marques, and R. Menezes, “Twinlike models for kinks, vortices, and monopoles," Physical Review D: Particles, Fields, Gravitation and Cosmology, vol. 96, no. 2, Article ID 025010, 9 pages, 2017.

[28] D. Bazeia, L. Losano, M. A. Marques, R. Menezes, and I. Zafalan, "Compact vortices," The European Physical Journal C, vol. 77, article no. 63, 2017.

[29] D. Bazeia, L. Losano, M. A. Marques, R. Menezes, and I. Zafalan, "First order formalism for generalized vortices," Nuclear Physics $B$, vol. 934, pp. 212-239, 2018.

[30] D. Bazeia, L. Losano, M. A. Marques, and R. Menezes, "Analytic vortex solutions in generalized models of the Maxwell-Higgs type," Physics Letters B: Particle Physics, Nuclear Physics and Cosmology, vol. 778, pp. 22-29, 2018. 
[31] R. Jackiw and E. J. Weinberg, "Self-dual Chern-Simons vortices," Physical Review Letters, vol. 64, no. 19, pp. 2234-2237, 1990.

[32] D. Bazeia, M. A. Marques, and R. Menezes, "Maxwell-Higgs vortices with internal structure," Physics Letters B, vol. 780, p. 485, 2018.

[33] E. Witten, "Superconducting strings," Nuclear Physics B, vol. 249, no. 4, pp. 557-592, 1985.

[34] T. Vachaspati and A. Achúcarro, "Semilocal cosmic strings," Physical Review D: Particles, Fields, Gravitation and Cosmology, vol. 44, no. 10, pp. 3067-3071, 1991.

[35] M. Hindmarsh, K. Rummukainen, and D. J. Weir, "New solutions for non-Abelian cosmic strings," Physical Review Letters, vol. 117, no. 25, Article ID 251601, 5 pages, 2016.

[36] A. Haber and A. Schmitt, "Critical magnetic fields in a superconductor coupled to a superfluid," Physical Review D: Particles, Fields, Gravitation and Cosmology, vol. 95, no. 11, Article ID 116016, 25 pages, 2017.

[37] M. Shifman, "Simple models with non-Abelian moduli on topological defects," Physical Review D: Particles, Fields, Gravitation and Cosmology, vol. 87, no. 2, Article ID 025025, 2013.

[38] A. J. Peterson, M. Shifman, and G. Tallarita, "Low energy dynamics of $U(1)$ vortices in systems with cholesteric vacuum structure," Annals of Physics, vol. 353, pp. 48-63, 2015.

[39] A. J. Peterson, M. Shifman, and G. Tallarita, "Spin vortices in the Abelian-Higgs model with cholesteric vacuum structure," Annals of Physics, vol. 363, pp. 515-532, 2015.

[40] D. Bazeia, M. A. Marques, and R. Menezes, "Magnetic monopoles with internal structure," Physical Review D: Particles, Fields, Gravitation and Cosmology, vol. 97, no. 10, Article ID 105024, 7 pages, 2018.

[41] G. Hooft, "Magnetic monopoles in unified gauge theories," Nuclear Physics B, vol. 79, no. 2, pp. 276-284, 1974.

[42] A. M. Polyakov, "Particle spectrum in quantum field theory," JETP Letters, vol. 20, no. 6, pp. 194-195, 1974.

[43] T. Vachaspati, "Dark strings," Physical Review D: Particles, Fields, Gravitation and Cosmology, vol. 80, no. 6, Article ID 063502, 2009.

[44] B. Hartmann and F. Arbabzadah, "Cosmic strings interacting with dark strings," Journal of High Energy Physics, vol. 2009, no. 7, article no. 68, 2009.

[45] A. J. Long, J. M. Hyde, and T. Vachaspati, "Cosmic strings in hidden sectors: 1 . Radiation of standard model particles," Journal of Cosmology and Astroparticle Physics, vol. 2014, no. 09, p. 30, 2014.

[46] A. J. Long and T. Vachaspati, "Cosmic strings in hidden sectors: 2. Cosmological and astrophysical signatures," Journal of Cosmology and Astroparticle Physics, vol. 2014, no. 12, article no. 40, 2014.

[47] A. E. Nelson and J. Scholtz, "Dark light, dark matter, and the misalignment mechanism," Physical Review D: Particles, Fields, Gravitation and Cosmology, vol. 84, no. 10, Article ID 103501, 2011.

[48] P. Arias, D. Cadamuro, M. Goodsell, J. Jaeckel, J. Redondo, and A. Ringwald, "WISPy cold dark matter," Journal of Cosmology and Astroparticle Physics, vol. 2012, no. 6, article no. 13, 2012.

[49] V. Silveira and A. Zee, "Scalar phantoms," Physics Letters B, vol. 161, no. 1-3, pp. 136-140, 1985.

[50] K. R. Dienes, C. Kolda, and J. March-Russell, "Kinetic mixing and the supersymmetric gauge hierarchy," Nuclear Physics B, vol. 492, no. 1-2, pp. 104-118, 1997.
[51] L. B. Okun, "Limits of electrodynamics: paraphotons?" Journal of Experimental and Theoretical Physics, vol. 56, p. 892, 1982, Journal of Experimental and Theoretical Physics, vol. 83, p. 892, 1982.

[52] P. Galison and A. Manohar, “Two Z's or not two Z's?” Physics Letters B, vol. 136, no. 4, pp. 279-283, 1984.

[53] B. Holdom, “Two U(1)'s and $\epsilon$ charge shifts," Physics Letters B, vol. 166, pp. 196-198, 1986.

[54] P. Arias and F. A. Schaposnik, "Vortex solutions of an Abelian Higgs model with visible and hidden sectors," Journal of High Energy Physics, vol. 2014, no. 12, article no. 11, 2014.

[55] P. Arias, E. Ireson, C. Núñez, and F. Schaposnik, "N=2 SUSY Abelian Higgs model with hidden sector and BPS equations," Journal of High Energy Physics, vol. 1502, no. 2, article no. 156, 2015.

[56] A. Haber and A. Schmitt, "New color-magnetic defects in dense quark matter," Journal of Physics G: Nuclear and Particle Physics, vol. 45, no. 6, Article ID 065001, 2018.

[57] S. Tulin and H.-B. Yu, "Dark matter self-interactions and small scale structure," Physics Reports, vol. 730, pp. 1-57, 2018.

[58] D. Budker, D. DeMille, D. F. Jackson Kimball, A. Derevianko, and C. W. Clark, "Search for new physics with atoms and molecules," Reviews of Modern Physics, vol. 90, Article ID 025008, 2018.

[59] M. N. Chernodub and A. S. Nedelin, "Pipelike current-carrying vortices in two-component condensates," Physical Review D: Particles, Fields, Gravitation and Cosmology, vol. 81, no. 12, Article ID 125022, 2010.

[60] M. Estrader, A. López-Ortega, S. Estradé et al., "Robust antiferromagnetic coupling in hard-soft bi-magnetic core/shell nanoparticles," Nature Communications, vol. 4, no. 1, article no. 2960, 2013.

[61] T. Okada, Y. González-Alfaro, A. Espinosa et al., "Magnetic and electronic properties of bimagnetic materials comprising cobalt particles within hollow silica decorated with magnetite nanoparticles," Journal of Applied Physics, vol. 114, no. 12, Article ID 124304, 2013.

[62] E. Mefford and G. T. Horowitz, "Simple holographic insulator," Physical Review D: Particles, Fields, Gravitation and Cosmology, vol. 90, no. 8, Article ID 084042, 2014.

[63] S. I. Finazzo and R. Rougemont, "Thermal photon, dilepton production, and electric charge transport in a baryon rich strongly coupled QGP from holography," Physical Review D: Particles, Fields, Gravitation and Cosmology, vol. 93, no. 3, Article ID 034017, 2016.

[64] R. H. Hobart, "On the instability of a class of unitary field models," Proceedings of the Physical Society, vol. 82, no. 2, pp. 201-203, 1963.

[65] G. H. Derrick, "Comments on nonlinear wave equations as models for elementary particles," Journal of Mathematical Physics, vol. 5, pp. 1252-1254, 1964. 

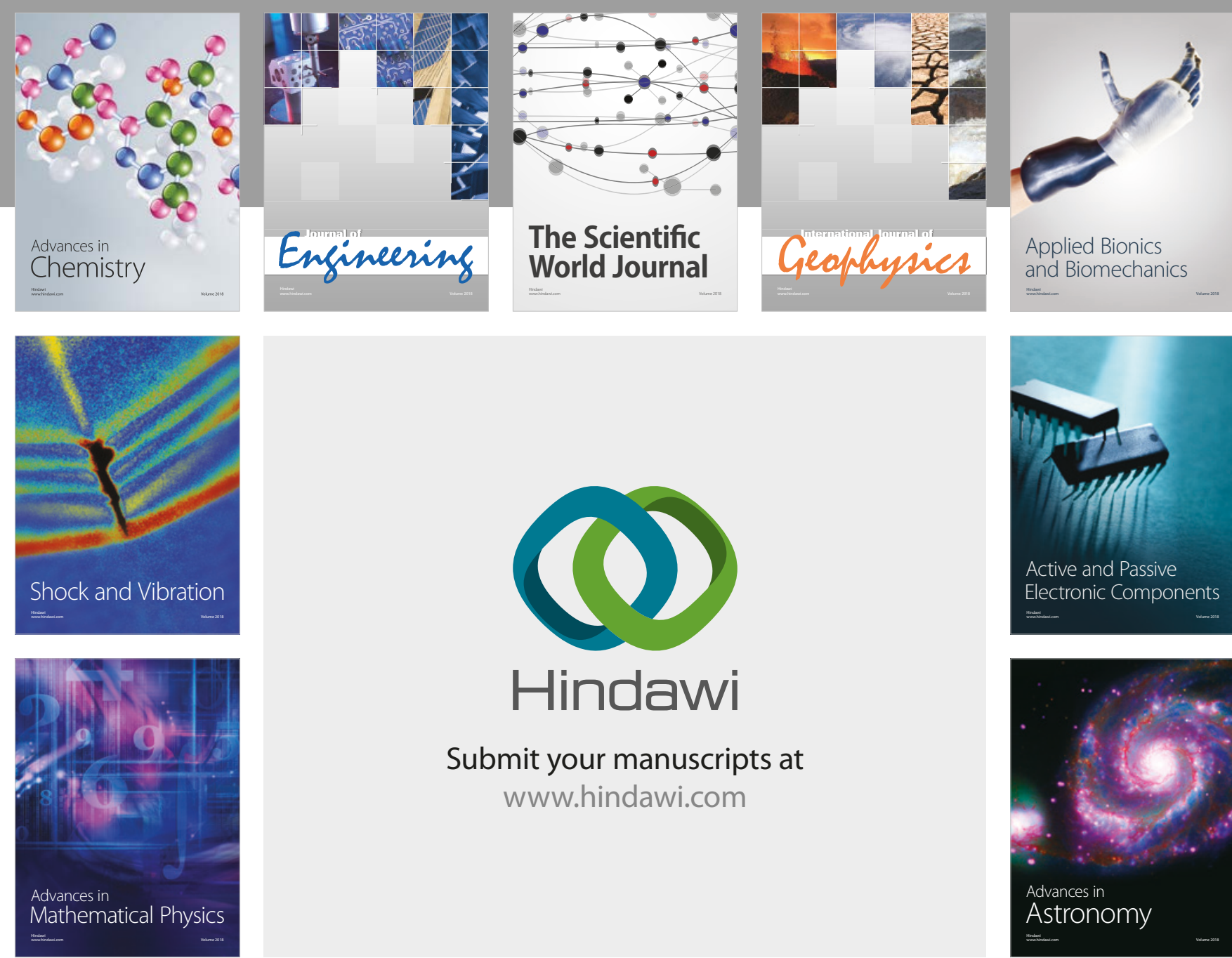

Submit your manuscripts at

www.hindawi.com

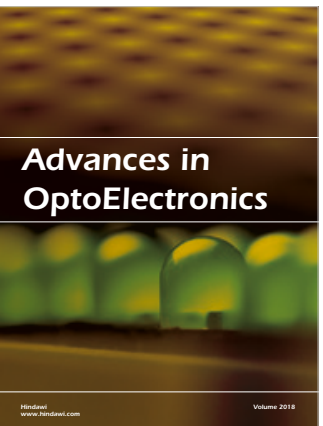

\section{Rotcting Machinery}
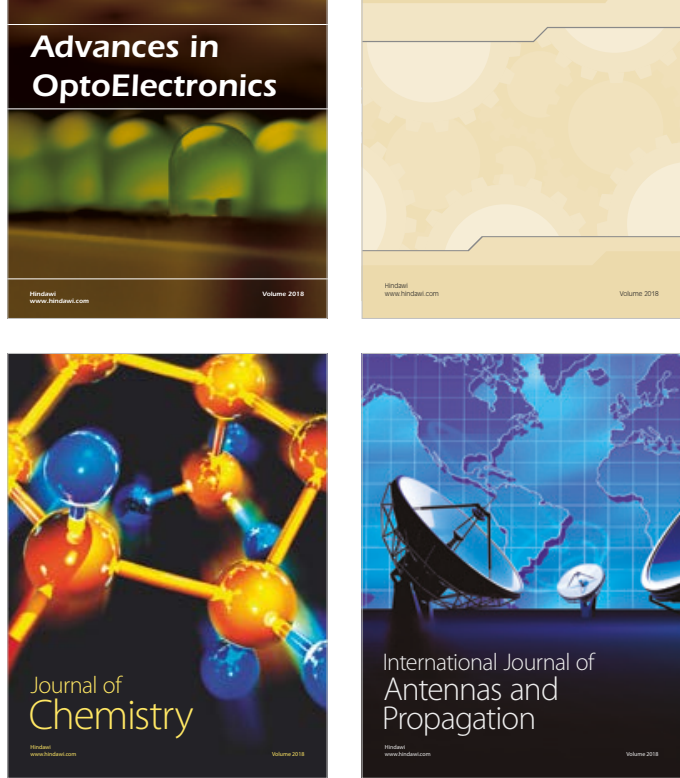

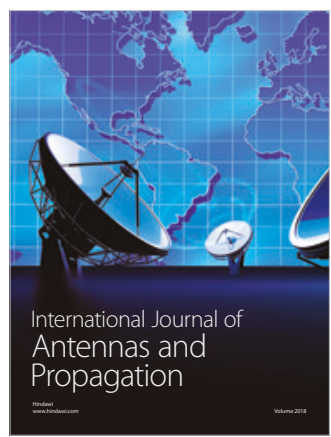

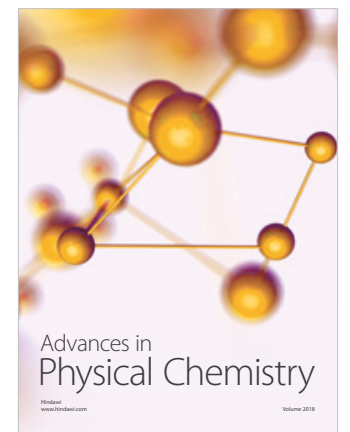

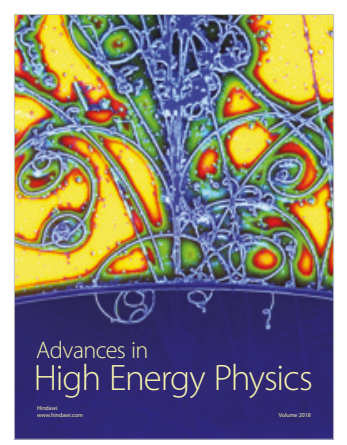

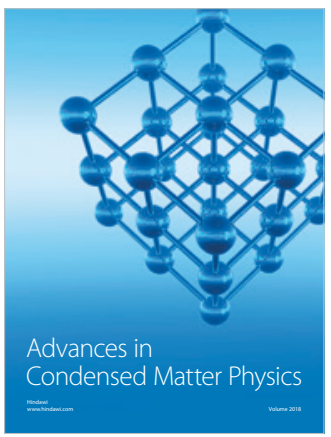

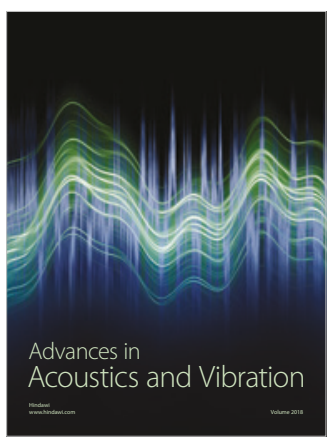

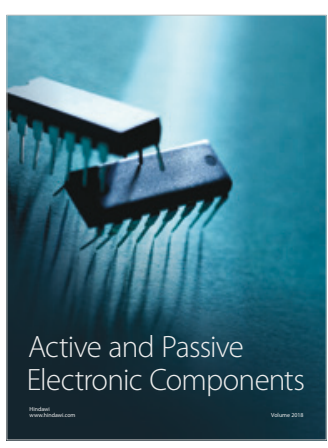
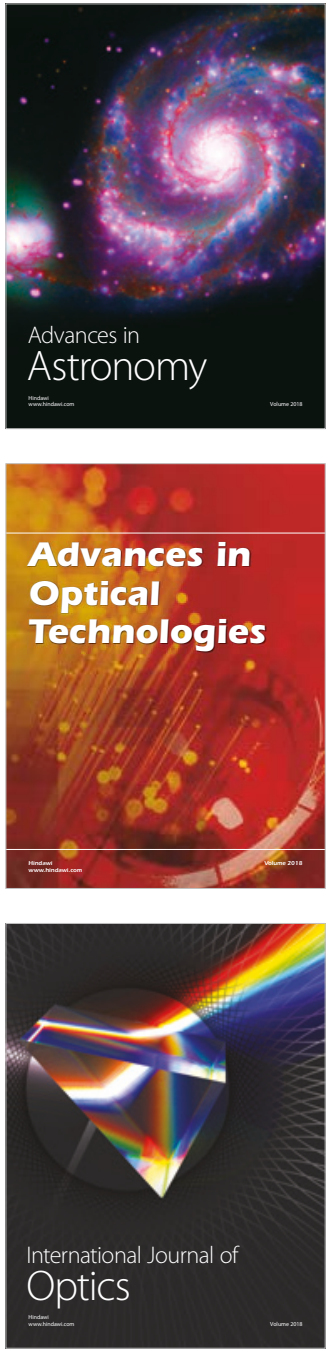